\title{
A Composite Heterostructure Mesh-shaped Patch Antenna Based on Left Handed Material
}

\author{
Ji-jun Wang, Zhi-pan Zhu*
}

Department of Physics, Jiangsu University, Zhenjiang, 212013, China

\begin{abstract}
In this paper, a composite heterostructure mesh-shaped patch antenna based on left handed material (LHM) is presented. The method of finite difference time domain (FDTD) is used. The results show that electromagnetic wave resonance occurs near $4.52 \mathrm{GHz}$, where the equivalent permittivity and permeability of composite material are both negative. The composite antenna's gain improves $9.047 \mathrm{~dB}$, its return loss reduces $20.26 \mathrm{~dB}$ compared to the conventional antenna's ones. The results indicate that this composite patch antenna system can reduce return loss of the antenna and increase the gain obviously.
\end{abstract}

Index Terms: LHM; Heterostructure; patch antenna; Return loss; Gain.

(C) 2014 Published by MECS Publisher. Selection and/or peer review under responsibility of the Research Association of Modern Education and Computer Science

\section{Introduction}

Left handed material (LHM) is a novel periodic artificial material with simultaneously negative values of dielectric permittivity and magnetic permeability. As early as 1968, Vselageo investigated the abnormal phenomenon of the LHM in theory [1]. When the electromagnetic (EM) wave propagates through the material, the electric field, magnetic field and wave vector of the electromagnetic wave would form a left-handed triad. In 2000, the first artificial LHM was fabricated by Smith and his co-workers by combining SRRs and continuous wires [2]. The success in fabrication opened up the possibility for applying LHM's abnormal EM characteristics in optical and electromagnetic areas.

The heterostructure is composed of two different photonic crystals. The aberrance in the interface of heterostructure will create localized mode and transmission mode of electromagnetic wave [3-5]. The property of heterostructure can be applied in patch antenna widely.

The paper employs FDTD method to analyze a composite heterostructure mesh-shaped patch antenna based on LHM, and its performance parameters are attained by simulation. The refraction index of the medium is extracted from its $\mathrm{S}$ parameters in order to validate availability of the structure. Then, the performance of this composite LHM patch antenna is analyzed by its performance parameters.

* Corresponding author. Tel.: 86 18796022027; fax: 8651188791128

E-mail address: zhuzhipan888@163.com 


\section{FDTD equations}

Macroscopic Maxwell equations are a set of basic equations to dominate the electromagnetic phenomena, which can be written in differential form, as well as in integral form. The two curl equations in Maxwell equations is the differential form of Faraday's law of electromagnetic induction and the Ampere's law. FDTD equations use the two curl equations as the starting point; calculate electromagnetic field problems directly in time domain.

Maxwell curl equations:

$$
\begin{aligned}
& \nabla \times \vec{H}=\vec{J}+\frac{\partial \vec{D}}{\partial t} \\
& \nabla \times \vec{E}=-\vec{J}_{m}-\frac{\partial \vec{B}}{\partial t}
\end{aligned}
$$

The relationship between electromagnetic fields and currents as follows:

$$
\begin{aligned}
& \vec{D}=\varepsilon \vec{E}, \quad \vec{B}=\mu \vec{H} \\
& \vec{J}=\sigma_{\varepsilon} \vec{E}, \quad \vec{J}_{m}=\sigma_{m} \vec{H}
\end{aligned}
$$

where $\vec{H}, \vec{E}, \vec{D}$ and $\vec{B}$ is the magnetic field, electric field strength, electric displacement vector and magnetic induction, respectively; $\vec{J}_{\text {and }} \vec{J}_{m}$ are the current density and magnetic flux density; $\varepsilon, \mu$ is the medium permeability and permittivity; $\sigma_{e}, \sigma_{m}$ is the medium of electrical conductivity and magnetic resistivity, corresponding to power loss and magnetic loss of material.

\section{Computing model of the patch antenna}

In the simulation of many algorithms of patch antenna, because the FDTD method has many advantages [68], it can be used to simulate the complex patch antenna commendably. Maxwell equations can be transformed into scalar field model by calculating in rectangular coordinate system, and then numerical difference coefficient in the second rank precision is employed to replace differential quotient. The differential equations are discreted in space-time using the method proposed by Yee, and the patch antenna is made meshed. We assume $\Delta x, \Delta y$ are space steps towards $\mathrm{x}$, y direction, respectively, $\Delta t$ is time step, then we can get difference equations in scalar field model. In transverse electric (TE) mode, Maxwell equations can be transformed into FDTD equations in iteration formulation:

$$
E_{x}^{n+1}(i, j)=E_{x}^{n}(i, j)+\frac{H_{z}^{n+\frac{1}{2}}\left(i, j+\frac{1}{2}\right)-H_{z}^{n+\frac{1}{2}}\left(i, j-\frac{1}{2}\right)}{\Delta y} \cdot \frac{\Delta t}{\varepsilon(i, j)}
$$




$$
\begin{aligned}
& E_{y}^{n+1}(i, j)=E_{y}^{n}(i, j)+\frac{H_{z}^{n+\frac{1}{2}}\left(i+\frac{1}{2}, j\right)-H_{z}^{n+\frac{1}{2}}\left(i-\frac{1}{2}, j\right)}{\Delta x} \cdot \frac{\Delta t}{\varepsilon(i, j)} \\
& H_{z}^{n+\frac{1}{2}}(i, j)=H_{z}^{n-\frac{1}{2}}(i, j)+\frac{E_{x}^{n}\left(i, j+\frac{1}{2}\right)-E_{x}^{n}\left(i, j-\frac{1}{2}\right)}{\Delta y} \cdot \frac{\Delta t}{\mu}-\frac{E_{x}^{n}\left(i+\frac{1}{2}, j\right)-E_{x}^{n}\left(i-\frac{1}{2}, j\right)}{\Delta x} \cdot \frac{\Delta t}{\mu}
\end{aligned}
$$

where, $\mathrm{n}$ is refer to cell number, and $\mathrm{i}, \mathrm{j}$ refer to two-dimensional coordinate. In order to ensure a steady iterative solution, $\Delta x, \Delta y, \Delta t$ must be selected to meet the stability condition necessarily[9]:

$$
\Delta t \leq \frac{1}{c \sqrt{(\Delta x)^{-2}+(\Delta y)^{-2}}}
$$

For the transverse magnetic (TM) mode, it can also get similar formula on Hx, Hy, Ez.

In the calculation procedure, we used perfectly matched layer (PML) boundary conditions in the X, Y direction [8]. Taking the Gauss pulse as the excitation source for its smoothness in the time domain, and the bandwidth is easy to choose. The electric field Ez vector under the micro strip on the excitation plane is:

$$
E z(t)=\exp \left[-\frac{\left(t-t_{0}\right)^{2}}{T^{2}}\right]
$$

The parameters are: $T=40 \Delta t, t_{0}=110 \Delta t$, where $\Delta t, t_{0}$ and $\mathrm{T}$ are time increment step, time delay, and half-width Gauss pulse. Its frequency ranges from 0 to $14.99 \mathrm{GHz} .4000$ time steps are chosen. The active patch antenna structure is calculated by the FDTD numerical method.

The geometry structure of composite heterostructure mesh-shaped patch antenna based on LHM is shown in Fig. 1 (a): the dimension of the patch antenna is $360 \mathrm{~mm} \times 360 \mathrm{~mm} \times 10 \mathrm{~mm}$, there are four layer substrates with $360 \mathrm{~mm}$ long and $360 \mathrm{~mm}$ wide, The first and the third layer of the substrate have the same thickness and relative permittivity, which are $3 \mathrm{~mm}$ and 2 . The second and the fourth layer have the same thickness and relative permittivity, which are $2 \mathrm{~mm}$ and 10 . The mesh-shaped radiating patch is etched on the top of the first layer substrate. Outside frame of the mesh-shaped patch is $340 \mathrm{~mm}$ long and $4 \mathrm{~mm}$ wide, the width of mesh line is $2 \mathrm{~mm}$, and their interval D1, D2 are $42 \mathrm{~mm}, \mathrm{D} 3, \mathrm{D} 4$ are $29 \mathrm{~mm}$. Two metal straps with the size 360 $\mathrm{mm} \times 6 \mathrm{~mm}$ are etched outside the mesh-shaped patch. Square SRRs and helices are inset the meshes of meshshaped patch alternately, and heterostructure is formed. Fig. 1 (b) shows the view of the helix, the width of helices D1 is $2.5 \mathrm{~mm}$, the distance between edges of two adjacent helices D2 is $2.5 \mathrm{~mm}$, the minimum radius R1 is $2.5 \mathrm{~mm}$, the radius R2 is $10 \mathrm{~mm}$, and R3 is $12.5 \mathrm{~mm}$. Fig. 1 (c) shows the view of the square SRR, opening of the square SRR is $3 \mathrm{~mm}$, the outside side length is $20 \mathrm{~mm}$, width is $2 \mathrm{~mm}$, the inner side length is $10 \mathrm{~mm}$, width is $2 \mathrm{~mm}$. The distance between the centers of adjacent helix and square SRR is $31 \mathrm{~mm}$. The second and the forth layer substrates have the same structure, which is shown in Fig. 2 (a). Square SRRs and E-shaped SRRs are etched on the top of the second and the fourth layer substrates alternately. Fig. 2 (b) shows the view of the Eshaped SRR, opening of the E-shaped SRR is $3 \mathrm{~mm}$, the outside side length is $20 \mathrm{~mm}$, and line width is $2 \mathrm{~mm}$. The distance between the centers of adjacent square SRR and E-shaped SRR is $31 \mathrm{~mm}$. Eight thin metal straps 
with the size $320 \mathrm{~mm} \times 5 \mathrm{~mm}$ are etched among square SRRs and E-shaped SRRs with the interval D7 = 28mm, D8 $=59 \mathrm{~mm}$. The structure of helices and square SRRs on the third layer substrate is the same as the first layer's ones, which is shown in Fig. 3. Eight thin metal straps of $320 \mathrm{~mm} \times 5 \mathrm{~mm}$ are etched among helices and square SRRs, and the interval D9 is $28 \mathrm{~mm}$, D10 is $59 \mathrm{~mm}$. In the bottom of the fourth layer substrate, there is a metal earth place shown in Fig. 4, which side is $360 \mathrm{~mm}$ long and $40 \mathrm{~mm}$ wide. Five $260 \mathrm{~mm} \times 40 \mathrm{~mm}$ metal straps are distributed symmetrically in the frame of metal earth place, and the interval is $15 \mathrm{~mm}$. The excitation source is Gaussian discrete source, fed by a micro strip whose width is $4.7 \mathrm{~mm}$, length is $10 \mathrm{~mm}$. Fig. 5 shows the view of every layer structure combination.

The conventional patch antenna without LHM is shown in figure 6, which dimension is $360 \mathrm{~mm} \times 360 \mathrm{~mm} \times$ $10 \mathrm{~mm}$, and the relative permittivity 10 . The mesh-shaped radiating patch is etched on the top of the substrate.

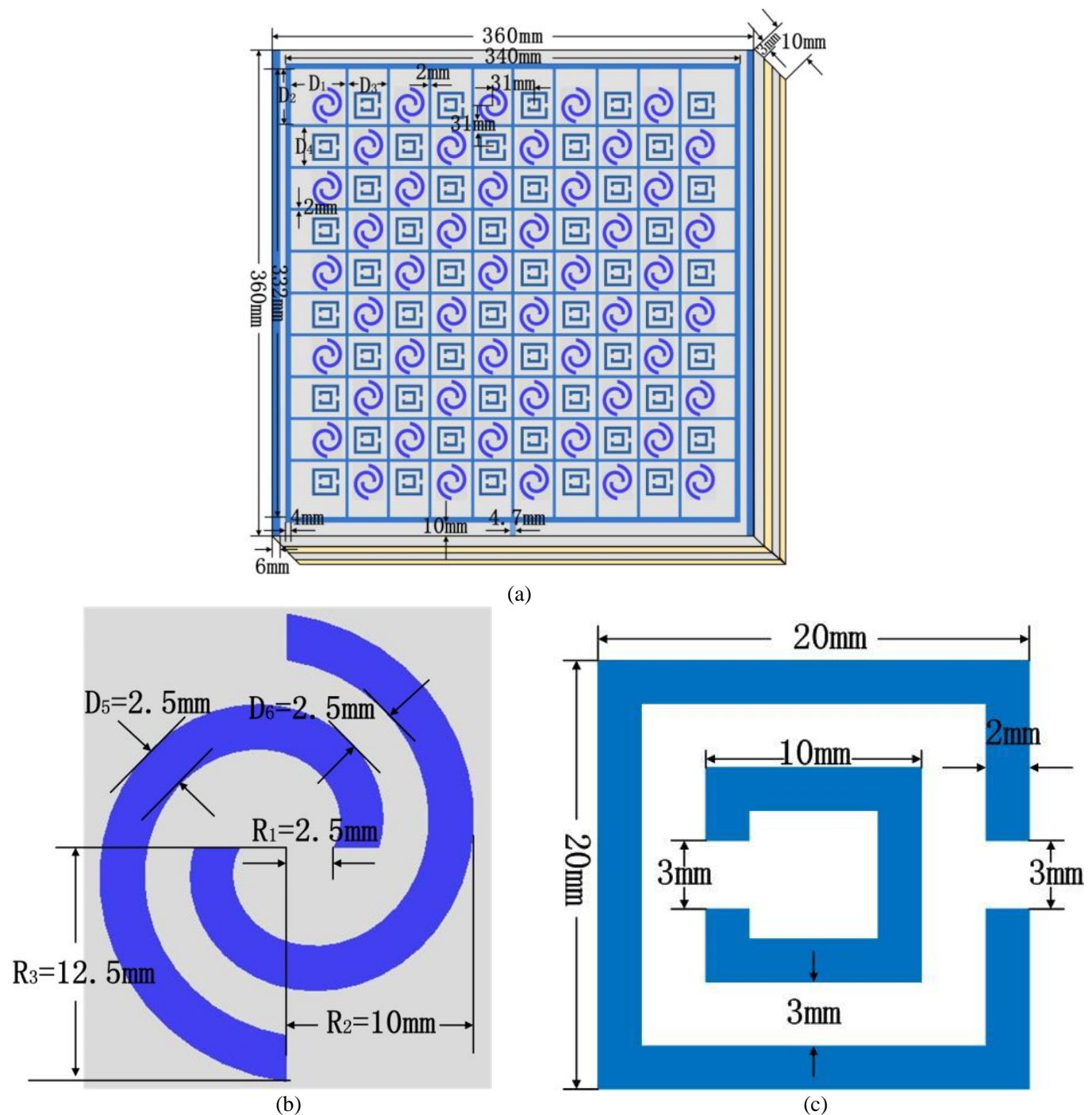

Fig.1. A top view of: (a) composite heterostructure mesh-shaped patch antenna based on LHM and (b) the helix (c) square SRR 


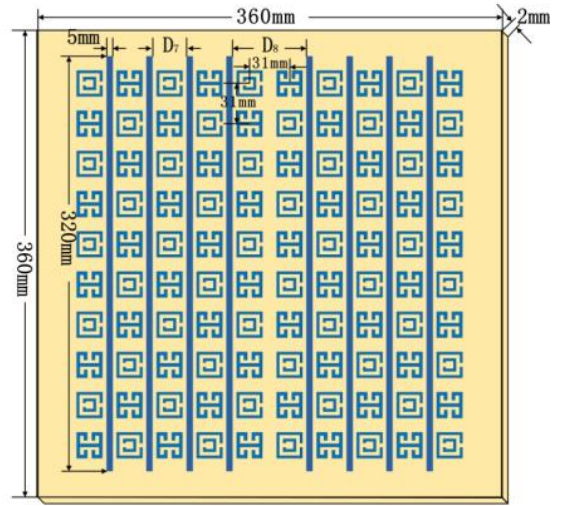

(a)

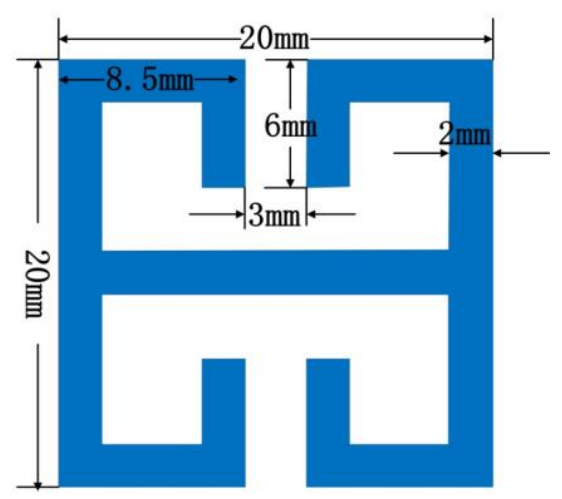

(b)

Fig.2. A top view of: (a) the second and the fourth layer substrates and (b) E-shaped SRR

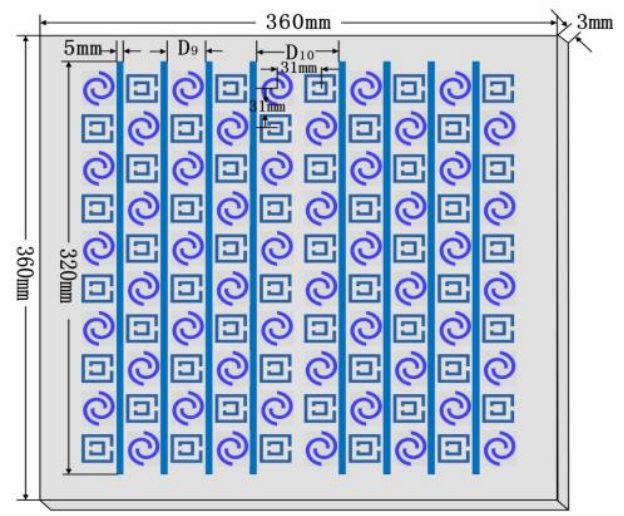

Fig.3. A top view of the third layer substrate

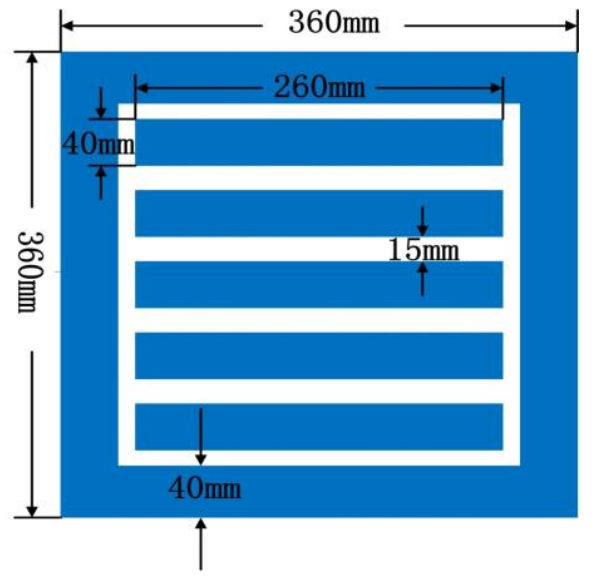

Fig.4. A view of metal earth place 


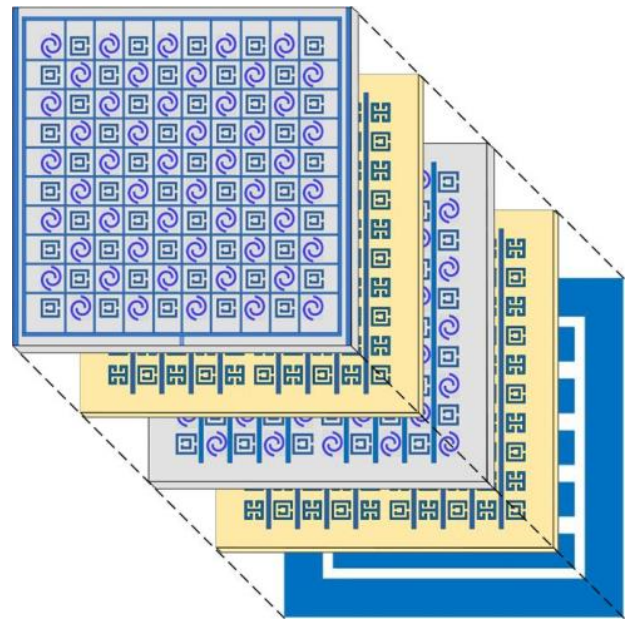

Fig.5. A view of every layer structure combination

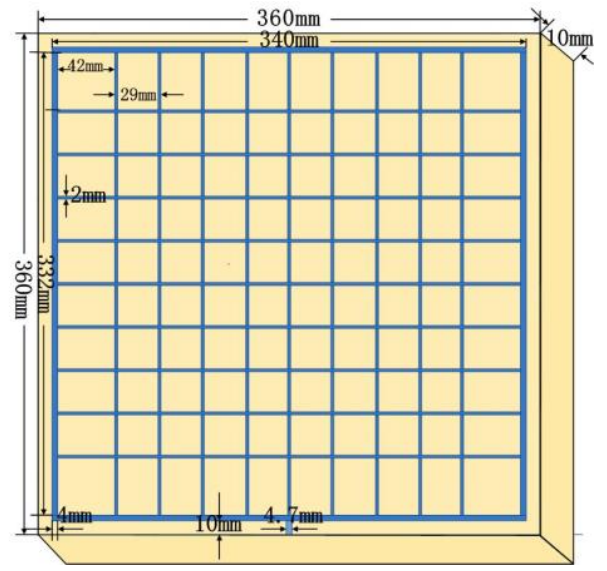

Fig.6. A top view of conventional patch antenna

\section{Simulation results and analysis}

The FDTD simulator is used to analyze the above composite heterostructure mesh-shaped patch antenna based on LHM and conventional patch antenna. The corresponding return loss (s11) and gain for both composite LHM patch antenna and conventional patch antenna are obtained, which are shown in Fig. 7 and Fig. 8.

It can be seen from Fig. 7 that the composite LHM patch antenna has a better return loss (s11), that's -32.60 $\mathrm{dB}$ at the frequency of $4.52 \mathrm{GHz}$, compared to the conventional patch antenna, $20.26 \mathrm{~dB}$ lower than the conventional one which gets $-12.34 \mathrm{~dB}$ at $4.55 \mathrm{GHz}$, so the composite LHM can improve the antenna's matching condition.

Narrow bandwidth is a major disadvantage of microstrip patch antenna. From Fig. 7, it is found that bandwidth of the composite LHM antenna is $1.06 \mathrm{GHZ}$ at $4.52 \mathrm{GHz}$, and adds 44 times compared to bandwidth of the conventional antenna, $0.024 \mathrm{GHZ}$ at $4.55 \mathrm{GHz}$. The results indicate that the bandwidth of composite LHM patch antenna is enlarged obviously, which improves patch antenna's property extremely. 


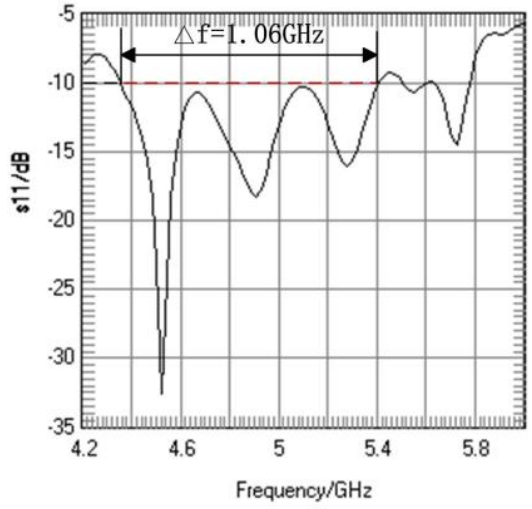

(a)

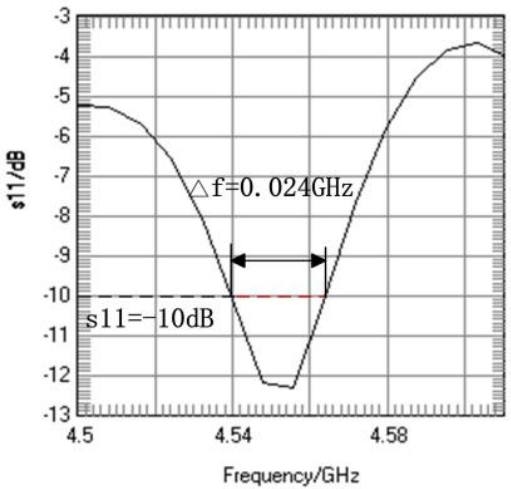

(b)

Fig.7. Return loss of: (a) composite heterostructure patch antenna based on LHM and (b) conventional patch antenna

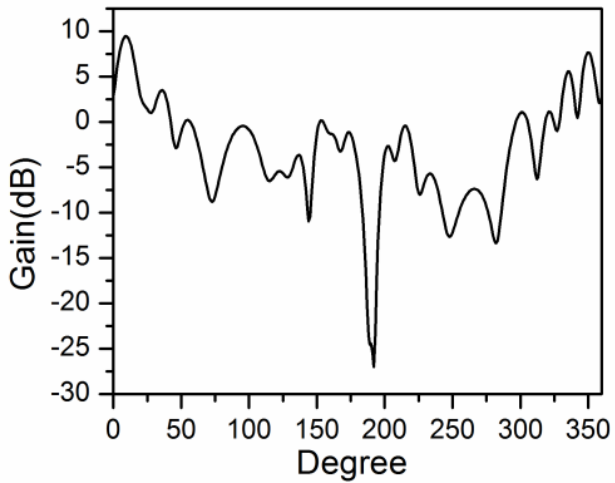

(a)

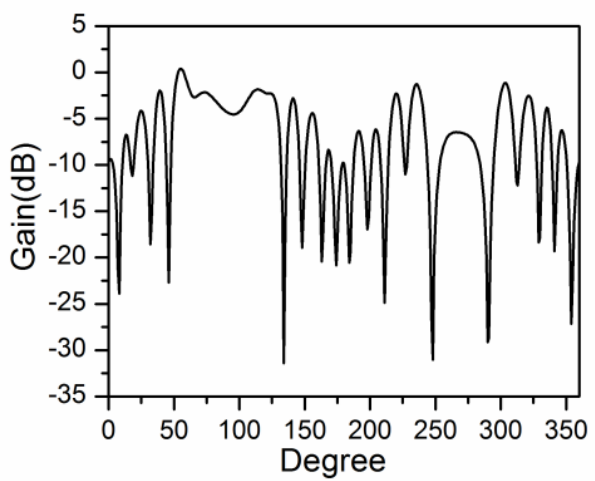

(b)

Fig.8. Gain of: (a) composite heterostructure patch antenna based on LHM and (b) conventional patch antenna

In the Fig. 8, we can find that the conventional antenna's maximum gain is $0.397 \mathrm{~dB}$ at $4.55 \mathrm{GHz}$, while the composite LHM's one is $9.444 \mathrm{~dB}$ at $4.52 \mathrm{GHz}$, which adds 23.8 times compared to the conventional antenna's one, and improves $9.047 \mathrm{~dB}$, showing that the composite LHM can improve patch antennas' gain obviously. The simulated results are listed in Table 1.

Table 1 Parameters of antennas with and without LHM

\begin{tabular}{cccccc}
\hline & $\begin{array}{c}\text { Resonant } \\
\text { frequency }(\mathrm{GHz})\end{array}$ & $\begin{array}{c}\text { Return loss } \\
(\mathrm{dB})\end{array}$ & Bandwidth (s11=-10dB) & $\begin{array}{c}\text { VSWR } \\
\text { gaximum }(\mathrm{dB})\end{array}$ \\
\hline composite LHM patch antenna & 4.52 & -32.60 & $\begin{array}{c}23.45 \% \\
(1.06 \mathrm{GHz} / 4.52 \mathrm{GHz})\end{array}$ & 1.048 \\
conventional patch antenna & 4.55 & -12.34 & $0.53 \%$ & 1.637 & 0.397 \\
\hline
\end{tabular}




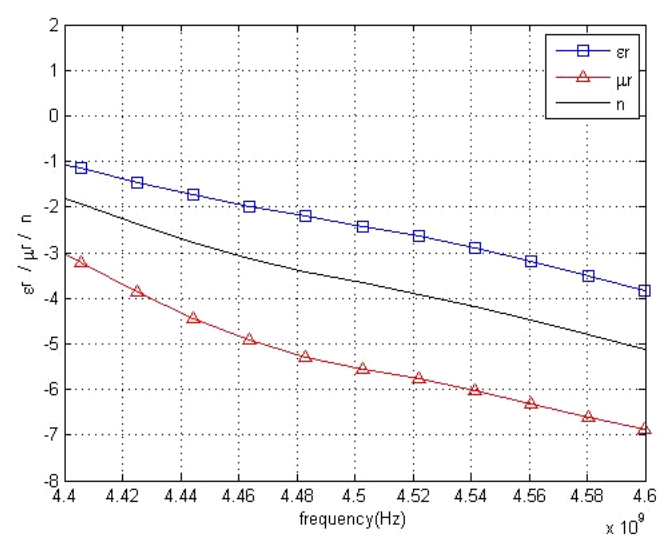

(a)

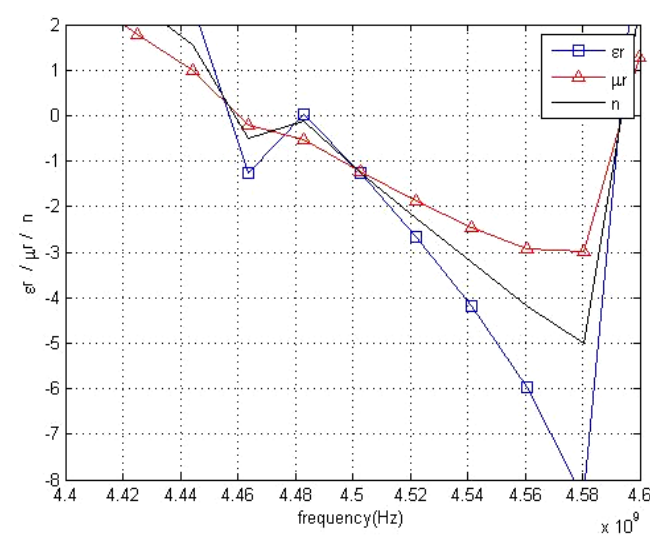

(b)

Fig.9. Equivalent permittivity $\varepsilon_{r}$, permeability $\mu_{r}$ and refraction index n of: (a) the first layer and the third layer substrate based on LHM and (b) the second and fourth layers substrates based on LHM

It can be easily found that the patch antenna presents lower return loss and higher gain by adding the LHM. This is clear from the following theoretical point of view: Because periodic square SRRs, helices, E-shaped SRRs and metals strips are added on the substrates, the negative permittivity and negative permeability can simultaneously appear in a certain frequency range. Thus, on the one hand, this paper proposes that metal straps are etched on the substrate to improve the structure's capacitance, and the added electric field will induce electricity through the patches, so an obvious electric resonance is created. On the other hand, periodic square SRRs, helices or E-shaped SRRs are etched on the substrate's surface so as to improve the structure's inductance, so an obvious magnetic resonance can be attained. When the above two negative parameters' area intersect, a double negative area is formed.

In order to validate the structure, we can use the $S$ parameters, including return loss (or: reflection coefficient) s11 and transmission coefficient s21 to extract the every layer composite structure's equivalent permittivity $\varepsilon_{r}$ and permeability $\mu_{r}$ by Nicolson Ross Weir (NRW) method [9-11]. The extracted results are shown in Fig.9. It can be seen that every layer composite structure's equivalent permittivity $\varepsilon_{r}$ and equivalent permeability $\mu_{r}$ are negative at $4.50 \mathrm{GHz}-4.58 \mathrm{GHz}$. According to $\varepsilon_{r}, \mu_{r}$, the refraction index $\mathrm{n}$ is calculated, and they are also negative, those are $\mathrm{n} 1$ (refraction index of the first layer composite structure) $=\mathrm{n} 3$ (refraction index of the third layer composite structure $)=-3.9$, and $\mathrm{n} 2$ (refraction index of the second layer composite structure) $=\mathrm{n} 4$ (refraction index of the fourth layer composite structure $)=-1.1$. These results indicate that the introduction of square SRRs, E-shaped SRRs, helices and metal straps to the patch antenna may result in equivalent negative

$\varepsilon_{r}$ and negative $\mu_{r}$. According to the above characteristics, it is explained that the negative-refraction dielectric can enhance the EM wave's tunnel effect [9], and the boundary plane between the positive refraction and negative refraction dielectric (the plane between the free space and composite structure) accord with the surface wave of the EM wave's tunnel traverse model [12]. These surface waves propagate through the boundary plane according to the evanescent waves' coupling effect. The power density near the boundary plane increase rapidly [10], indicating that the equivalent negative refraction structures have the effect of amplifying evanescent waves, so the transmission of surface waves in these models can be enhanced obviously. This phenomenon can improve the antenna's gain, and improve the system's matching condition. 
What's more, after embedding the heterostructure into the antenna, due to the electromagnetic waves' highly localized effect caused by aberrance between two heterostructures, the electromagnetic energy near a frequency range would get a high gain.

\section{Conclusion}

A composite heterostructure mesh-shaped patch antenna based on left handed material (LHM) is fabricated by assembling helices, square SRRs, E-shaped SRRs and metals strips on the substrates of conventional antennas. According to our simulation and analysis, we find that this composite patch antenna system can improve patch antenna's property extremely. On the one hand, the electromagnetic wave resonance occurs near $\mathrm{f}=4.52 \mathrm{GHz}$, and the equivalent permittivity and permeability of every layer composite material are negative. The electromagnetic wave's tunnel effect and evanescent waves' enhancing effect are formed, which can improve the localization extent of electromagnetic wave's energy apparently. Such effects can improve the antenna's radiation gain and its matching condition. Therefore, the radiation power coupled into the free space can be enhanced. On the other hand, the heterostructure is embedded into the antenna. Due to the electromagnetic waves' highly localized effect caused by aberrance of heterostructure, the electromagnetic energy near a frequency range would get a high gain. So the antenna's performance can be improved. Due to these advantages, the use of this composite patch antenna can be extended to mobile communication, satellite communication, aviation, etc.

\section{Reference}

[1] V. G. Vesolago, The electrodynamics of substances with simultaneously negative values of $\varepsilon$ and $\mu$, Soznet Physics Uspekh, 1968, 10, 509 - 514.

[2] D. R. Smith and N. kroll, Negative refraction index in left handed materials, Phys. Rev. Let., 2000, 85, 2933-2936.

[3] L. L. Lin and Z. Y. Li, Interface states in photonic crystal heterostructures, Phys Rev B., 2001, 63, 033310-1 033310-4.

[4] Y. S. Zhou, B. Y. Gu and F. H. Wan, Guided modes in photonic crystal heterostructure composed of rotating non-circular air cylinders in two-dimensional lattices, J Phys: Condens. Matter, 2003, 15, 4109 4118.

[5] Y. S. Zhou, B. Y. Gu and F. H. Wang, Photonic band-gap structures and guide modes in two-dimensional magnetic photonic crystal heterostructure, Eur Phy J B, 2004, 37, 293 299.

[6] S. W. Yang, Y. K. Chen and Z. P. Nie, Simulation of time modulated linear antenna arrays using the FDTD method, Progress in Electromagnetics Research, 2009, 98, 175-190.

[7] A. C. M. Austin, M. J. Neve and G. B. Rowe, Modeling propagation in multifloor buildings using the FDTD method, IEEE Trans. Antennas and Propagation, 2011, 59, 4239-4246.

[8] X. T. Dong, X. S. Rao, Y. B. Gan, B. Guo, and W. Y. Yin, Perfectly matched layer absorbing boundary condition for left handed materials, IEEE Microwave and Wireless Components letters, 2004, 14, 301-303.

[9] Z. M. Zhang and C. J. Fu, Unusual photon tunneling in the presence of a 1ayer with a negative refractive index, Phys. Rev. Let., 2002, 80, 1097 - 1099.

[10] J. B. Pendry, Negative Refraction Make a Perfect Lens, Phys. Rev. Let., 2000, 85, 3966.

[11] R. W. Ziolkowski, Design, Fabrication, and Testing of Double Negative Metamaterials, IEEE Trans. Antennas and Propagation, 2003, 51, 1516-1528.

[12] I. V. Shadrivov, A. A. Sukhorukov and Y. S. Kivshar, Nonlinear surface waves in left-handed materials, Phys. Rev. E, 2004, 69, 016617-1 016617-9. 


\section{Authors Profiles}

Ji-Jun Wang received the B.S. degree in physics from Tianjin University, Tianjin, China, in 1986, the M.S. in power electronics and power drives from Jiangsu University, Zhenjiang, China, in 2003, and Ph.D. degrees in optical engineering from Nanjing University of Science and Technology, Nanjing, China, in 2008, respectively. In 2004, he participated in the project of the National Natural Science Foundation of China (NSFC), (60208004). He is currently a professor of Department of Physics, Jiangsu University. His research interests include laser physics, design and application of antennas, theory and design of optoelectronic devices and optical communication.

Zhi-Pan Zhu received the B.S. degree in applied physics from Suzhou University of science and technology, Suzhou, China, in 2011. He is currently working toward the M.S. degree in physical electronics at Jiangsu University, Zhenjiang, China. His current research interests include design and application of antennas, microwave and optical communication application and design of optoelectronic devices.

How to cite this paper: Ji-jun Wang, Zhi-pan Zhu,"A Composite Heterostructure Mesh-shaped Patch Antenna Based on Left Handed Material", IJWMT, vol.4, no.1, pp.10-19, 2014.DOI: 10.5815/ijwmt.2014.01.02 